\title{
A linear stability theory on time-invariant and time-dependent spatial domains with symmetry: the drop splash problem
}

\author{
Rouslan Krechetnikov \\ In memory of Professor Jerrold E. Marsden, who carried passion for mechanics, symmetry, and weather through \\ his life
}

\begin{abstract}
This work is a combination of physical and analytical considerations of linear stability pictures on time-invariant and time-dependent spatial domains with symmetry. The discussion is offered in the context of the Rayleigh-Taylor instability (of a fluid interface accelerated in the direction of a heavier phase) applied to the drop splash problem, which provides a natural ground for developing stability theory on time-dependent spatial domains with $O(2)$ symmetry. The peculiarity of the underlying linear model common in a number of other interfacial instabilities - linear oscillator $f_{t t}+a(k) f=0$ in the wavenumber $k$-space - allows one to establish a direct correspondence between stability pictures on time-invariant and time-dependent spatial domains. The stability analysis also leads to a notion of frustration in (linear) stability patterns.
\end{abstract}

\section{CONTENTS}

1. Introduction 47

2. Stability picture on time-invariant domains with $O(2)$ symmetry 50

3. Stability picture on time-dependent domains with $O(2)$ symmetry 57

4. Conclusions 63

Appendix A. Richtmyer-Meshkov instability with a nearly impulsive acceleration 64 References

\section{Introduction}

1.1. General context. The presented work is focused on two aspects in the general stability theory - the relationship between stability pictures on time-invariant and timedependent spatial domains with symmetries and frustration phenomena. This study was triggered by both experimental observations of frustration phenomena in the drop splash

1991 Mathematics Subject Classification. 76, 35.

Key words and phrases. Drop splash, Rayleigh-Taylor instability. 
problem [38], cf. figure 1(b), and the relevant theoretical finding of the situation when several wavenumbers can be excited with the same growth rate $[36,37]$. The time-dependence and symmetry of the crown geometry in the drop splash problem justifies the focus of this work. As motivated by the physical considerations relevant to the drop splash phenomena $[36,37]$, we will analyze problems, which linearized dynamics is governed by the oscillator-type model,

$$
f_{t t}+a(t ; k, \mu) f=0,
$$

in the wavenumber $k$-space; here $t$ is time and $\mu$ is a set of parameters. The model (1.1) is common in the linear description of a number of classical interfacial instabilities [16].

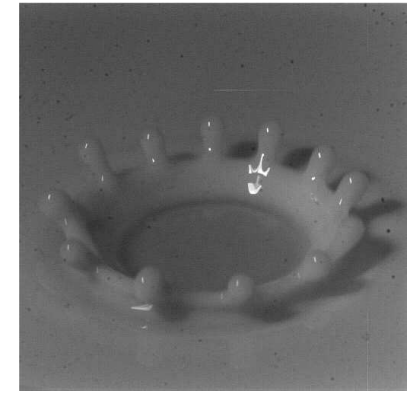

(a) Regular crown.

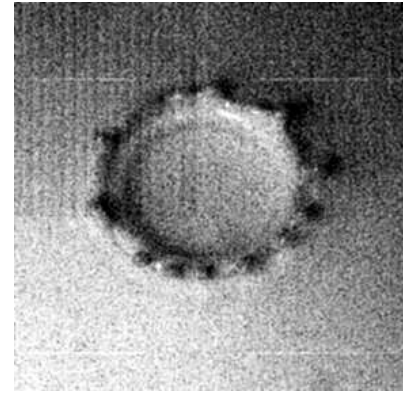

(b) Frustrated crown.

FIgURE 1. Patterns observed in the drop splash problem [38].

Besides the drop splash problem, there is a large number of other phenomena on spatial time-dependent domains, e.g. transport-reaction processes - crystal growth, metal casting, gas-solid reaction systems - as well as classical electromagnetic cavity resonators with moving walls $[\mathbf{6}, \mathbf{1 4}, \mathbf{4 8}, \mathbf{3 9}, \mathbf{5 2}]$, quantum mechanical problems [15], fluid motion $[58,27,40,44,61,59]$, fluid-structure interaction [24], and formation of patterns and shapes in biology $[\mathbf{2 9}, \mathbf{4 2}, \mathbf{3 5}]$. The most common approach [2] to analyze problems on time-dependent spatial domains is to map them onto a new fixed in space domain. The simplest case corresponds to the situation when the boundaries are deformed slowly compared to the period of the most unstable mode. In this case a solution can be constructed by multiple scale asymptotic methods [48]. Another common approach for studying stability and long-time behavior of these problems is by energy methods, as done in the context of fluid mechanics for the Navier-Stokes equations [61], Cosserat fluids [59], wave equations $[\mathbf{1 4}, \mathbf{4 8}]$, and the nonlinear beam equation $[\mathbf{2 5}]$. In the cases when there is a well-defined map from the time-dependent spatial domain onto the one fixed in space, the linearized stability problem is then about stability of a time-dependent base state, which usually reduces to the analysis of problems with non-autonomous linear operators, even if the original operator is autonomous. The most well-studied case corresponds to time-periodic bases states [12] as it allows straightforward application of Floquet theory [26]. In general, however, the arsenal of methods which allow one to get analytical results for problems with non-autonomous operators is very limited. Namely, one has to recourse either to energy methods [31] or to computation of Lyapunov exponents [22, 23] of the propagator $\Phi_{\left[t, t_{0}\right]}$, which evolves the solution $u(x, t)=\Phi_{\left[t, t_{0}\right]} u\left(x, t_{0}\right)$ from the initial condition $u\left(x, t_{0}\right)$.

As for the frustration phenomena, their discussion in the context of other problems is implicit in standard stability analyses. For example, even in the following standard solution 
representation for the scalar field [46]

$$
\psi(x, t)=\operatorname{Re}\left\{\psi_{1} e^{i k_{1} x}+\psi_{2} e^{i k_{2} x}\right\}+\ldots,
$$

where $\psi_{1}$ and $\psi_{2}$ are the amplitudes of the primary modes corresponding to single wavenumber $k_{1}$ - and $k_{2}$-patterns, one can think of a spatial phase-shift between these modes, which is due to random initial conditions, to be hidden in the expression of the mode amplitudes $\psi_{1}, \psi_{2}$. Growth rates corresponding to the wavenumbers $k_{1}$ and $k_{2}$ are different, in general. The present work is based on the recently found [37] peculiar dispersion relation in the context of interfacial instabilities, which allows the existence of the same growth rates for different single-wavenumber patterns.

1.2. Motivation. As mentioned above, one of the motivations for this study stems from the phenomena of drop splashing on a liquid film. The complexity of this classical hydrodynamics problem, which is known since the classical works [66], suggests that a simple model capable of accurate prediction of the crown evolution may not be feasible. Therefore, this problem generated many conjectures about the mechanisms of the crown formation (see the discussions in [38] and $\S 3.1$ ), which have not yet helped one to clarify its basic dynamical features. Here, based on recent experimental observations [38] and stability theory of accelerated curved interfaces [36, 37], we propose a model which helps one to extract some of the fundamental components of the drop splash phenomena during linear stages of the crown formation. This approach proves to be useful for explaining not only the physical mechanisms behind the crown formation, but also for qualitative understanding of the complex dynamics in this problem. Effectively, we will replace the crown dynamics in the physical space, which requires a partial differential equation model to be accounted for, with a simpler model in the wavenumber space.

As known from experimental observations [38], in certain ranges of physical parameters the crown structure changes from regular (single wavenumber) to irregular with intermittent frustration phenomena as the height $H$ of the drop release increases ${ }^{1}$ for fixed fluid properties - density $\rho$ and surface tension $\sigma$ - and a ratio of the drop diameter to the pre-existing film thickness $d / h$. Except for the frustration regime, the number of spikes increases with $H$. In the regular regime the number and amplitude of spikes are stable for a fixed $H$.

It was also found [38] that in certain regimes the number of spikes and thus the regularity of the crown structure - regular, frustrated, and irregular - are generally dictated by its evolution at early times during which it is called ejecta. The latter fact means that the wavelength of the instability along the crown rim $k(t)$ normalized by the rim radius $R(t)$ - or, equivalently, the number of spikes - is set by the linear instability mechanisms. Notably, this behavior is in contrast to other systems on time-dependent domains such as the appearance of new stripes in the skin pattern of the Pomacanthus fish as it ages [35]. The conditions for this peculiar dynamics will be given in $\S 3$ when developing stability theory of the drop splash phenomena on time-evolving domains. In general, in the course of ejecta evolution, the rim shown in figure 2(a) experiences substantial time-dependent acceleration $g(t)$; the geometry of the rim - its radius $R(t)$ and radius of curvature $a(t)-$ is time-dependent as well. An important question to tackle is what kind of restrictions does the fact that the number of spikes stays the same over the time of ejecta evolution impose on the stability picture?

\footnotetext{
${ }^{1}$ Alternatively, the control parameter is the Weber number of the drop $W e_{\text {drop }}=\rho v_{0}^{2} d / \sigma$; here $\rho$ is the density and $\sigma$ surface tension of the fluid, $d$ the drop diameter, and $v_{0}=\sqrt{2 g H}$ the impact velocity of the drop released from the height $H$ in the gravity field $g$.
} 
Moreover, this problem is notable because the base state - flat rim in figure 2(a) has continuous Galilean symmetry, while the perturbation imposed on it has a discrete symmetry in figure $1(\mathrm{a})$, namely $2 \pi$-periodicity and reflection symmetry which altogether yield the $O(2)$-symmetry.

1.3. Paper outline. The paper is organized as follows. First, in $\S 2$, we focus on the case of time-invariant spatial domains using a motivating physical example of a liquid rim developed in $\S 2.1$ and which stability is analyzed in $\S 2.2$. This leads to a concept of frustration phenomena in the linear stability theory on spatial domains with $O(2)$ symmetry. In $\S 3$, we extend this discussion to time-dependent domains and compare the outcome with the results of $\S 2$. In conclusion, $\S 4$, we pose questions requiring further exploration.

\section{Stability picture on time-invariant domains with $O(2)$ symmetry}

2.1. Physically motivated model problem: time-independent liquid rims. Here we will develop a model based on the physics of the drop splash problem, which will be used in the subsequent stability analysis on time-independent spatial domains. In this section we neglect by the time-dependence of the base state and domain in the drop splash problem, which is relevant to certain regimes of impact, e.g. on deep liquid films as shown in figure 2(b). Namely, we will develop a linear stability model for liquid rims when perturbations imposed on flat rim, cf. figure 2(a), lead to instability, cf. figure 2(b).

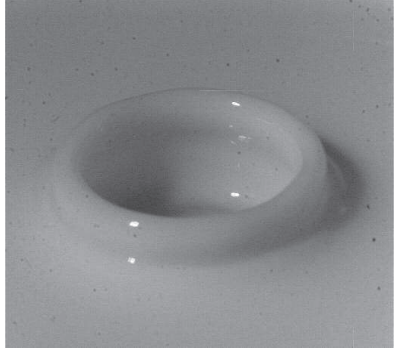

(a) Stable rim (milk).

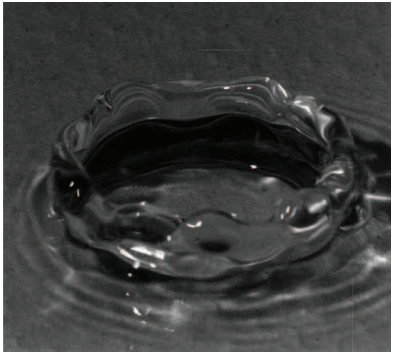

(b) Unstable rim (water).

FIGURE 2. Rims as a basis for the model (2.6).

In the context of our model (1.1) to be justified below, the bifurcation parameter $\mu$ is the Bond number,

$$
B o=\rho g_{0} L^{2} / \sigma,
$$

which is a measure of surface tension effects versus inertia; here $g_{0}$ is a characteristic value of the rim acceleration and $L$ is an appropriate length scale, which is set to a characteristic value $R_{0}$ of the rim radius $R(t)$. Based on the discussion in the introduction, one can expect that the crown structure is a function of all four parameters: $B o, a(t), R(t)$, and $g(t)$, which are considered time-independent in this section. Due to non-zero acceleration $g$, the rim should be subject to the Rayleigh-Taylor instability [37].

Let us first briefly remind the classical Rayleigh-Taylor instability $[49,60,16]$ of a flat semi-infinite interface between a vacuum and a heavy fluid, which is accelerating with $g=$ const. This instability is described [16] in the irrotational inviscid approximation 


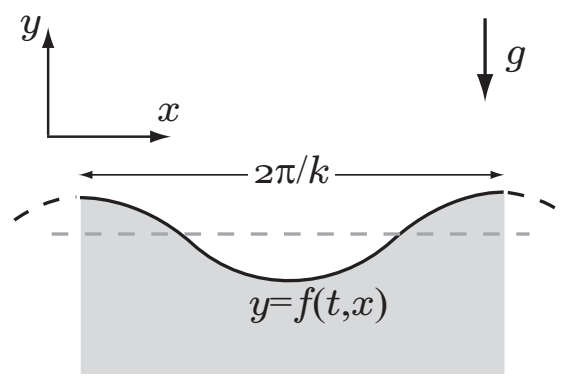

FIGURE 3. Accelerating two-dimensional interface; the coordinate system is accelerating with the flat interface base state.

by the following non-dimensional equation for the evolution of the interfacial disturbance $f(t ; k)=\int f_{k}(t) e^{i k x} \mathrm{~d} k$ in the $k$-wavenumber space:

$$
\frac{\mathrm{d}^{2} f_{k}}{\mathrm{~d} t^{2}}+|k|\left[\frac{k^{2}}{B o}-g\right] f_{k}=0,
$$

where the Bond number is defined in (2.1) and $g$ is the non-dimensional acceleration, which can be taken as $g=1$ since $g=$ const. As one can observe from this equation, the disturbance will grow if the wavenumbers satisfy $k^{2}<g B o$, i.e. this is a long-wave instability since the short waves are stabilized by surface tension effects. The physical meaning of the factor $|k|$ is the penetration of disturbances at the depth $\sim k^{-1}$ as dictated by the form of the velocity potential (i.e. the fluid velocity in the bulk is defined by $\boldsymbol{v}=$ $\nabla \phi)$

$$
\phi(t, x, y)=\int \phi_{k}(t) e^{i k x+|k| y} \mathrm{~d} k,
$$

as the solution of the following elliptic problem in the lower half-plane:

$$
\begin{gathered}
\Delta \phi=0, \\
|\nabla \phi| \rightarrow 0, y \rightarrow-\infty .
\end{gathered}
$$

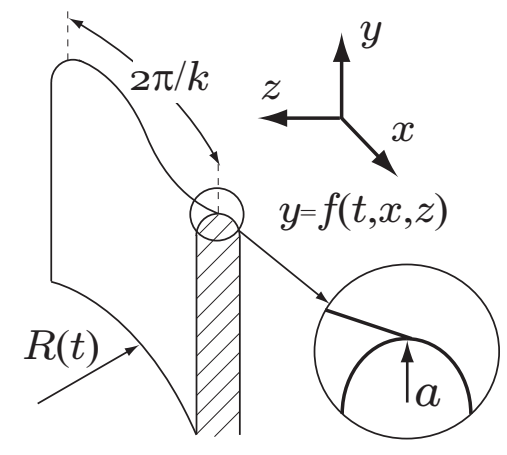

FIgURE 4. Perturbation along the rim in the three-dimensional $(x, y, z)$-space. 


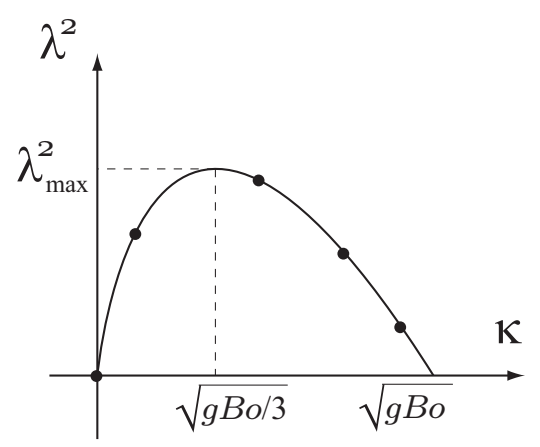

(a) $\lambda^{2}(\kappa), \kappa=\sqrt{k^{2}+n^{2}}$.

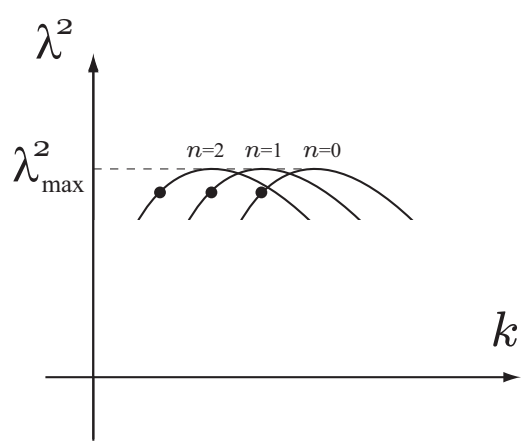

(b) $\lambda^{2}(k), k=\sqrt{\kappa^{2}-n^{2}}$.

FIGURE 5. Growth rate $\lambda$ behavior; curves correspond to $k \in \mathbb{R}$ and discrete points to $k \in \mathbb{Z}$.

In the three-dimensional case of liquid rims, cf. figure 4 with $R=\infty$, the velocity potential as a solution of $(2.4)$ is given by $[\mathbf{3 6}, \mathbf{3 7}]$

$$
\phi(t, x, y, z)=\sum_{n} \int \phi_{k n}(t) e^{i \frac{\pi n z}{a}} e^{i k x} e^{\sqrt{k^{2}+\frac{\pi^{2} n^{2}}{a^{2}}} y} \mathrm{~d} k .
$$

This solution should be contrasted with (2.3): the decay rate of the perturbation in the bulk is now defined by the depth $a$, i.e. the rim thickness, since $e^{\frac{i \pi n z}{a}}$ has the main variation for $n=1$ over the flow domain. Next, obviously $|k| \ll \pi / a$ and thus the decay rate is $\sim \pi / a$. This in turn affects the instability growth rate and the selection of the most unstable wavenumber, since the factor $|k|$ in (2.2) will be replaced with $a^{-1}$.

With an appropriate rescaling of the coordinates, the amplitude equation for $f_{k n}(t)$ reads

$$
\frac{\mathrm{d}^{2} f_{k n}}{\mathrm{~d} t^{2}}-\lambda^{2} f_{k n}=0, \lambda^{2}=\kappa\left[g-\frac{\kappa^{2}}{B o}\right],
$$

where $\lambda$ is the growth rate, $\kappa$ the two-dimensional wavenumber, i.e. $\kappa^{2}=n^{2}+k^{2}$. In the case when $k \in \mathbb{R}$, the solution in the physical space is represented by

$$
f(t, x, z)=\int_{\mathbb{R}_{n}} \sum_{n=-\infty}^{\infty} f_{k n}(t) e^{i[k x+\varphi(n)]} e^{i n z} \mathrm{~d} k,
$$

where wavenumber $k$ sets up the solution structure along the rim and $\varphi(n)$ is the phase corresponding to $n$.

\subsection{Linear stability and frustration phenomena.}

2.2.1. The concept of frustration in stability theory. As follows from (2.6), for $k \in \mathbb{R}$, the growth rate $\lambda$ can be depicted as in figure 5(a). Its maximum is achieved for $\kappa_{\max }=$ $\sqrt{g B o / 3}$ and equals to $\lambda_{\max }=\sqrt[4]{(4 / 27) g^{3} B o}$. Instability happens when $B o$ crosses its critical (for a given wavenumber $k$ ) value $B o_{c}=\kappa^{2} / g$; based on the behavior of the eigenvalues $\lambda$-they move along the imaginary axis, collide, and then split along the real axis in the complex plane - this is a steady-state Hamiltonian bifurcation. Since $\kappa^{2}=$ $k^{2}+n^{2}$, if $\kappa_{\max }^{2}=g B o / 3>n^{2}, n \in \mathbb{Z}$, then one can get several wavenumbers excited as in figure 5(b). The critical phenomena correspond to the ones shown in figure 6: for each $n$ there corresponds a critical curve $g B o=\kappa^{2}=k^{2}+n^{2}$ above which one gets instability, 
i.e. there exists $\lambda$ with $\operatorname{Re}(\lambda)>0$. By choosing $g$ Bo above critical value, one observes a range of unstable wavenumbers.

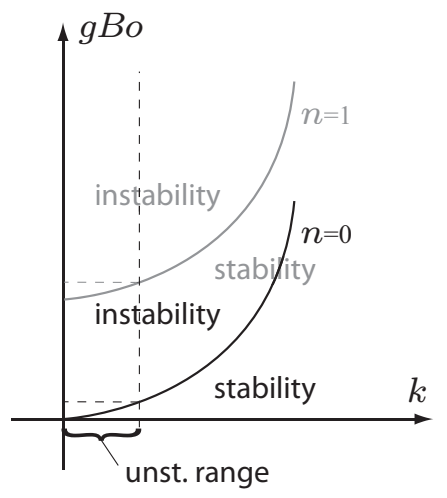

FIGURE 6. Critical phenomena and the range of unstable (excited) wavenumbers.

Next, let us restrict the solution (2.6) to a circular rim, cf. figure 7. In the case when $k \equiv m \in \mathbb{Z}$, which happens when the domain is circular thus confining the wavenumbers to be integers, the stability curves become discrete, cf. figure 5, and the solution in the physical space is represented by

$$
f(t, x, z)=\sum_{m, n=-\infty}^{\infty} f_{m n}(t) e^{i[m x+\varphi(n)]} e^{i n z},
$$

where $\varphi(n)$ is a random phase-shift. The case of existence of several critical wavenumbers found recently [37], cf. figure 5(b), means that the resulting pattern can consist of several single-wavenumber patterns with random phase-shifts between them, which brings us to the frustration picture in stability theory. While the notion of frustration may have not been introduced in the literature explicitly, it is implicit in a number of studies, e.g. refer to formula (2) in [46] for the scalar field

$$
\psi(x, t)=\operatorname{Re}\left\{\psi_{1} e^{i k x}+\psi_{3} e^{3 i k x}\right\}+\ldots,
$$

where $\psi_{1}$ and $\psi_{3}$ are the amplitudes of the primary modes corresponding to single wavenumber $k$ - and $3 k$-patterns; one can think of a spatial phase-shift between these modes to be hidden in the expression of the mode amplitudes $\psi_{1}, \psi_{3}$.

DEFINITION 1. Frustration picture in linear stability theory corresponds to the situation of a superposition of two or more single-wavenumber patterns with a random phaseshift between them.

Note that this definition allows the wavenumbers of single-wavenumber patterns to be the same, but as long as there are random phase-shifts between them, the resulting stability picture is frustrated.

The above stability analysis agrees qualitatively with the experimental observations of the number of spikes as a function of the release height $H$, cf. figure 6 in [38]: namely the number of spikes increases as a square root of the Bond number (up to a certain limit, when the number of spikes becomes so large that frustrated picture is indistinguishable from irregular one from a practical viewpoint). Figure 8 illustrates patterns formed for 


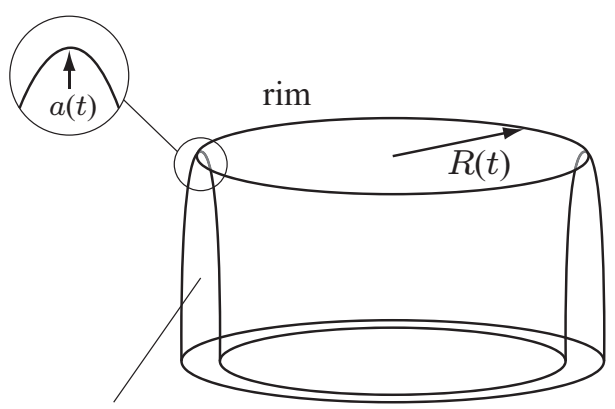

liquid sheet

FIGURE 7. Base state motivated by the rim in figure 2(b).

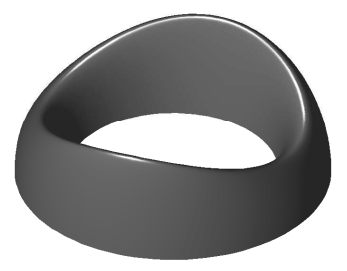

(a) $B o=12$.

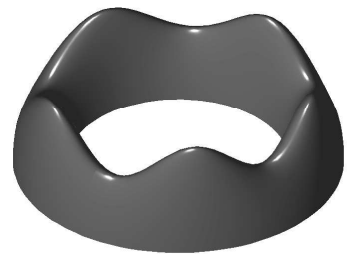

(d) $B o=75$.

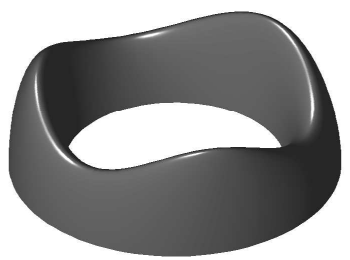

(b) $B o=27$.

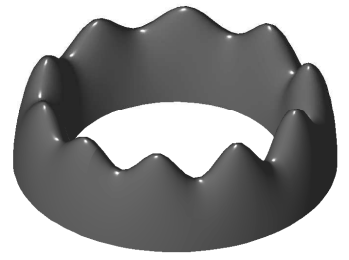

(e) $B o=300$.

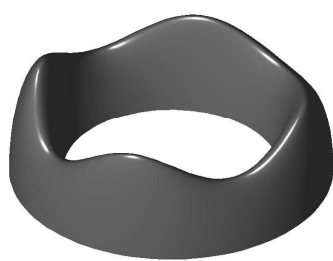

(c) $B o=48$

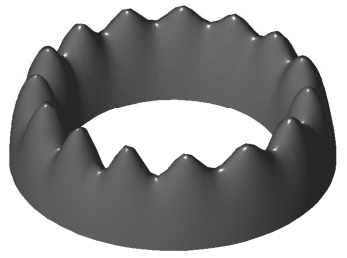

(f) $B o=675$.

FIGURE 8. Patterns predicted by the model (2.6): the case of 'resonant' Bond numbers producing integer values of $m$ ( $n$ is taken to be zero).

resonant Bond numbers (i.e. which satisfy $\kappa_{\max }^{2}=m^{2}+n^{2}=g B o / 3$ with $m$ and $n$ being integers), which agrees with the prediction based on the model (2.6).

In the case of non-resonant $B o$, cf. figure 9, the patterns exhibit partially merged fingers and the degree of their overlap depends on how close the Bond number is to the resonant value. The key question is at which Bond number does the transition between spike numbers take place? It is certainly dictated by linear amplification mechanisms as well as random processes, which are responsible for particular initial conditions. As it is easy to conclude, the transition occurs when the growth rates $\lambda_{1}$ and $\lambda_{2}$ of the neighboring integer wavenumbers $k_{1}$ and $k_{2}$, respectively, become equal. Strictly speaking, in this case there must be a frustration pattern with $k_{1}+k_{2}$ with a random phase shift. This motivates the introduction of the concept of "finite-time frustration" since for $\lambda_{1}$ close to $\lambda_{2}$ it takes $\sim\left|\lambda_{1}-\lambda_{2}\right|^{-1}$ time to 'win' for one of the single-wavenumber patterns.

2.2.2. Discussion. Frustration phenomena may also originate from other reasons even at the linear level of modeling, which can be appreciated with the help of the following 


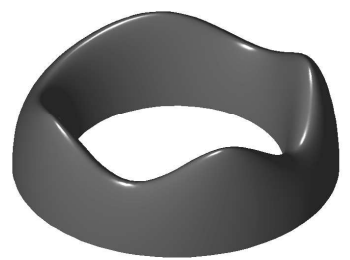

(a) $B o=58$.

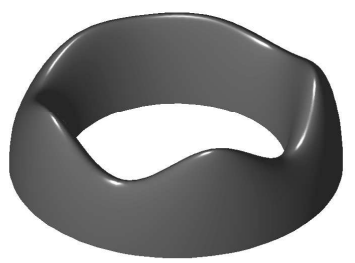

(b) $B o=60$.

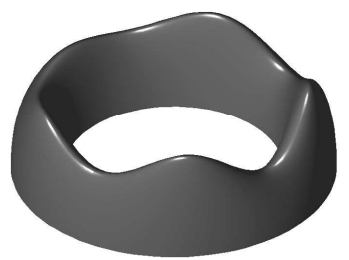

(c) $B o=61$.

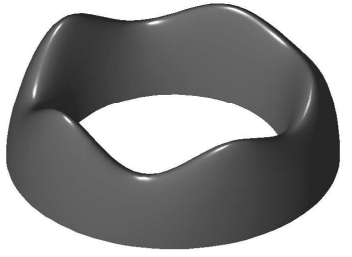

(d) $B o=62$

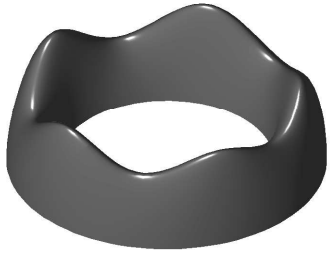

(e) $B o=65$.

FIGURE 9. Transitional patterns corresponding to the Bond numbers between the resonant values: transition from $m=4$ to $m=5$ ( $n$ is taken to be zero).

amplitude equation

$$
\frac{\partial u}{\partial t}=\left(B o-k_{0}^{2}\right) u+\frac{\partial^{2} u}{\partial x^{2}}-2 i k_{0} \frac{\partial u}{\partial x}, x \in[-R, R],
$$

where $B o$ stands for a bifurcation parameter. If the domain is finite, i.e. $R$ is bounded, then the critical "curve" is discrete, $B o=\left(\pi n R^{-1}-k_{0}\right)^{2}$ with $n \in \mathbb{Z}$, which is obviously generally non-symmetric with respect to $k_{0}$. As the domain size $R$ changes, the discrete critical set travels along the continuous critical curve corresponding to $R=\infty$. Thus, it is possible that at some value of the bifurcation parameter $B o$ above critical $B o_{c}$ there could be two or three excited critical wave-numbers. The case of two excited wave-numbers leads to frustration, while the case of three wave-numbers may lead to chaotic behavior, due to three-wave interaction [7] should nonlinearity be included in (2.10). The above argument is, of course, valid only if the instability wavelength is capable of adjusting itself to possible changes (in time) of the domain size. Therefore, another origin of frustration phenomena can be the dynamically changing size of the domain (i.e. expanding crown), $R(t)$, when the critical wavelength, which clearly is a function of $R$, cannot 'catch up' with the domain size changes, provided the instability characteristic time is slower than that of the evolution of $R(t)$.

At the nonlinear level, the presence of at least two competing spatial modes may lead to spatial chaos ${ }^{2}$, which may be responsible for irregular patterns observed in the late stages of the drop splash problem [38]. Classical problems, where spatial chaos was also found, are condensed matter systems where one of the periods is that of the crystal lattice, and the other period that of a modulated ordered structure [33]. There is nontrivial weakly nonlinear theory developed for such systems with spatial resonances and generalized to the case of two resonant wavenumbers, in particular $1: 2[\mathbf{3}], 1: 3$ [46], $1: n$ [46], and

\footnotetext{
${ }^{2}$ This is opposed to temporal chaos, which is a consequence of resonant effects between two or more competing frequencies.
} 


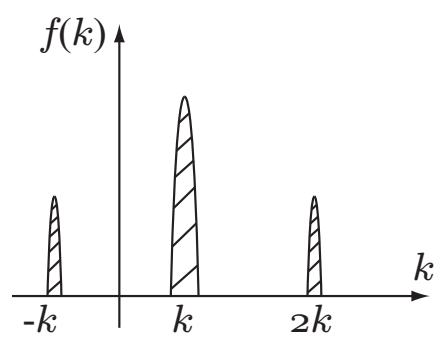

FIGURE 10. Mode-clustered form of the solution.

general rational ratio $m: n[\mathbf{1 0}]$; however in all these cases the growth rates are usually different [46]. As pointed out earlier, in the present work the growth rates of different single-wavenumber patterns are the same due to the peculiar dispersion relation (2.6).

Let us consider the case when there are two wavenumbers excited in (2.6), e.g. $k_{0}$ and $k_{1}$ corresponding to $n_{0}=0$ and $n_{1}=1$ respectively. In order to arrive at the weakly nonlinear description [18], one needs the growth rate to be positive but small $0<\lambda \ll 1$ and the range of excited (i.e. "unstable") wavenumbers to be asymptotically narrow, so that one can use the mode-clustered form of the solution $[18, \mathbf{1 9}, \mathbf{4 5}, \mathbf{6 4}, \mathbf{5 5}, \mathbf{5 6}, \mathbf{3 2}]$, cf. figure 10 , for each single-wavenumber pattern. Note that a random phase shift between patterns of different wavenumbers does not affect weakly nonlinear model for a single-wavenumber pattern; indeed

$$
f_{2 k} e^{2 i k(x-\phi)} f_{-k} e^{-i k(x-\phi)} \sim f_{2 k} f_{-k} e^{i k(x-\phi)},
$$

that is due to the mode-clustered form of the solution, the phases are locked to the phase of the main wavenumber $k$ because of nonlinear interactions.

In view of the symmetry of the circular rim, we consider a representation of the symmetry group $O(2)$, which is defined by the operations of rotation and reflection,

$$
\begin{aligned}
\left(f_{1}, f_{2}\right) & \rightarrow\left(e^{i k m \varphi} f_{1}, e^{i k n \varphi} f_{2}\right), 0 \leq \varphi<2 \pi, \\
\left(f_{1}, f_{2}\right) & \rightarrow\left(\bar{f}_{1}, \bar{f}_{2}\right),
\end{aligned}
$$

respectively, acting on complex vectors $\left(f_{1}, f_{2}\right) \in \mathbb{C}^{2} \simeq \mathbb{R}^{4}$. Here $\bar{f}$ is the complex conjugate of $f, m$ and $n$ are primes, and $k$ is the largest common divisor of the integer wavenumbers $k_{0}$ and $k_{1}$, respectively: note that wavenumbers are bound to be integers in our case due to circular domain. Identification of vector fields which are equivariant under (2.12) lead to the following normal form for the system based on the model (2.2) with $O(2)$ symmetry in the case when there are two single-wavenumber patterns excited with a random phase-shift between them

$$
\begin{aligned}
& \frac{\mathrm{d} f_{1}}{\mathrm{~d} t}=\lambda f_{1}+A\left(\left|f_{1}\right|^{2}+\left|f_{2}\right|^{2}\right) f_{1}+B\left|f_{1}\right|^{2} f_{1}, \\
& \frac{\mathrm{d} f_{2}}{\mathrm{~d} t}=\lambda f_{2}+A\left(\left|f_{1}\right|^{2}+\left|f_{2}\right|^{2}\right) f_{2}+B\left|f_{2}\right|^{2} f_{2},
\end{aligned}
$$

where $f_{1}, f_{2} \in \mathbb{C}, A, B \in \mathbb{C}, \lambda \in \mathbb{R}^{+}$. The solution in the physical space has the form ${ }^{3}$

$$
f(t, x, z) \simeq f_{1} e^{i k_{0}\left(x+\varphi_{0}\right)} e^{i n_{0} z}+f_{2} e^{i k_{1}\left(x+\varphi_{1}\right)} e^{i n_{1} z},
$$

\footnotetext{
${ }^{3}$ This is opposed to the standard case $[\mathbf{1 0}, \mathbf{1 1}]$, where random phases are not shown explicitly.
} 
where $\varphi_{0}, \varphi_{1} \in \mathbb{R}$ are random phases. However, once nonlinear stage in evolution is achieved (the appropriate time scales are determined by the coefficients $A$ and $B$ in (2.13)), the solution pattern will no longer be a simple superposition of single-wavenumber patterns with a random phase shift between them, as nonlinear dynamics generally changes this linear picture $[\mathbf{9}, \mathbf{3 4}, \mathbf{4 7}]$.

As easy to show, the use of polar $(r, \theta)$-coordinates, $f_{1}=r_{1} e^{i \theta_{1}}$ and $f_{2}=r_{2} e^{i \theta_{2}}$, decouples (2.13) into amplitude and phase equations, which is a consequence of the $S O(2)$ and $S(1)$ symmetries. Equations (2.13) have the following three types of solutions $\left(r_{1}, r_{2}\right)$ [11]: the trivial solution $(0,0)$, the traveling wave $(r, 0)$ or $(0, r)$, and the standing wave $(r, r)$. All other solutions are transient. The presence of two or more competing spatial modes may lead to spatial chaos, which is consistent with the observation of irregular patterns [38]. At the linear level, the existence of frustrated patterns - a superposition of two or more single-wavenumber modes with a random phase-shift between them - may also lead to irregularity if the experimental scatter is of the order of the pattern wavelengths, so that the frustration pattern is not identifiable. It is also important to keep in mind that even in the case when frustration patterns are identifiable, they exhibit randomness since the phase-shifts between its components are random as set-up by initial conditions. In general, the complex behavior of the nonlinear model $(2.13)$ is reasonably well understood $[\mathbf{1 0}, \mathbf{4 6}]$ and may be of qualitative guidance to the experimentally observed complex dynamics in the drop splash problem. The present study, however, is focused on the linear (short-time) dynamics as justified by the experimental observations that the spike structure set at early times (and thus linear stage) of evolution propagates to later times in the studied regimes [38].

\section{Stability picture on time-dependent domains with $O(2)$ symmetry}

The central theme of this section is establishing a connection between linear stability pictures on time-invariant and time-dependent domains: we focus here on the effects of unsteadiness and leave the additional frustration effects to future studies. As mentioned above, experimental observations [38] indicate that the number of spikes is generally constant over the course of the ejecta evolution (for the range of physical parameters studied in [38]), which leads us to the following conjecture.

CONJECTURE 1. In the framework of the models (2.6) and (3.1), the time-rate of evolution of acceleration and that of the geometry in (3.1) should be the same, i.e. $g(t) \sim$ $R^{-2}(t)$, in order to get a finite wavenumber instability.

In what follows, we will provide a theoretical basis for this conjecture. Namely, we will develop a model for the time-dependent base state - the rim - on which the instability develops leading to the crown formation ( $(3.1)$, and then analyze its stability $(\S 3.2)$.

3.1. Physically motivated model problem: time-dependent liquid rims. As depicted in figure 11, after the drop impact a thin layer of liquid is ejected from the preexisting liquid film, cf. figure 11(a), which then evolves into a crown, cf. figure 11(b), as the drop penetrates into the film. In the course of evolution, the interface first experiences a vary rapid acceleration as the film fluid is initially at rest and shortly after impact attains considerable velocity [38], and then deceleration as the retraction mechanisms due to surface tension slow down the ejecta (crown) growth. As argued in [38], the RichtmyerMeshkov instability [50] takes place during the first stage since the interface experiences a nearly impulsive acceleration (cf. Appendix), and the Rayleigh-Taylor instability during 
the later stages ${ }^{4}$. Also, due to high curvature of the interface near the tip, Rayleigh-Plateau mechanisms make the evolution of the Richtmyer-Meshkov and Rayleigh-Taylor instabilities very different from those for flat interfaces classically studied $[\mathbf{3 6}, \mathbf{3 7}]^{5}$, i.e. both growth rates and critical wavenumber selection are affected by the curvature effects as one can see from the dependence of the solution (2.5) on the curvature $a(t)$, which is of the order of the ejecta thickness.

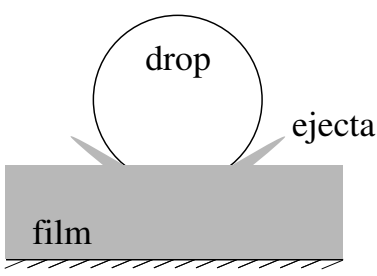

(a) Formation of ejecta.

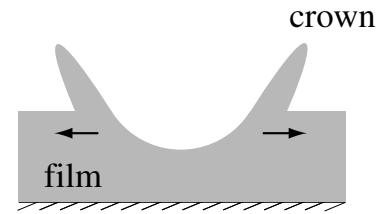

(b) Crown evolution.

FIGURE 11. Schematics of the crown formation in the drop splash on a liquid film.

In this section we provide a physically motivated analytical example of a time-dependent base state corresponding to the early stages of the crown formation in the drop splash on liquid films, which, to the author's knowledge has not been developed before. The previous analytical attempts to study the dynamics of the drop splash problem on liquid films were concerned with a depth of cavity by Engel $[\mathbf{2 0 , 2 1}]$ but not with the ejecta evolution, which is reflected in the simplifying geometric assumptions built in these works $[\mathbf{2 0 , 2 1 ]}$. The analogous geometric considerations were used in the work of Macklin and Metaxas [41], who neglected the crown rim thickness as well. Conservation laws were also utilized in the recent works by Trujillo and Lee [63] and Roisman and Tropea [53], which were concerned with late stages of the crown formation. Here we deal with the early stages of the crown formation, i.e. ejecta origin and evolution, since the regimes of interest [38] are those when the crown structure is dictated at these early times. The base state constructed below applies to certain regimes of the drop splash phenomena, e.g. impact on thick enough films

\footnotetext{
${ }^{4}$ We recall that the basic difference between the Richtmyer-Meshkov and the Rayleigh-Taylor instabilities is the sudden acceleration of the interface in the Richtmyer-Meshkov case as opposed to a constant acceleration in the Rayleigh-Taylor case. The former allows for the development of interfacial instability regardless of the direction of acceleration.

${ }^{5}$ The above understanding was developed just recently [38, 36, 37]. Earlier, Fullana and Zaleski [28] commented that the instability mechanism remained undetermined and put forward the idea that the crown formation in problems like drop splashing on thin films is due to the Rayleigh-Plateau capillary instability of the cylindrical rim that develops at the end of the planar sheet. Their analytical study, based on one-dimensional macroscopic balance, showed that "the growing cylindrical end rim does not typically break into droplets for moderate wavelength", i.e. the authors could not detect an instability with their model. However, the Rayleigh-Plateau mechanism may be dominant in certain situations of the drop splash phenomena such as splashing on very thin films [68]. In the review paper by Yarin [67], only two competing theories are mentioned: (a) the capillary instability of the rim, considered as a toroidal thread, and (b) the bending instability of the toroidal rim. Also, Gueyffier and Zaleski [30] speculated that a possible instability mechanism in a drop splashing on thin liquid films might be of the Richtmyer-Meshkov type, but did not provide any evidence to support or refute the conjecture. To date the drop splash problem has been studied most extensively by experimental means. In particular, a nice parametric study of identification of various regimes of splashing, i.e. with or without a crown formation, was done in [51]. Details of the crown were measured in [8] in the case of an impact on thin film for the drop Weber numbers $W e_{\mathrm{drop}}=\rho v_{0}^{2} d / \sigma=O\left(10^{3}\right)$. Drop splashing on very thin films was also studied in [65].
} 


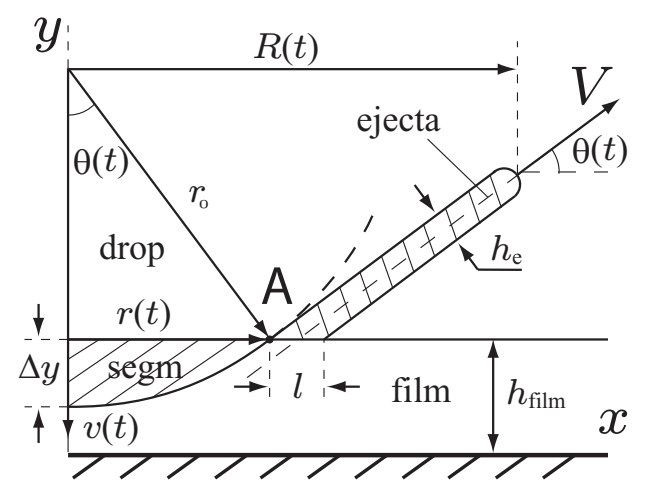

FIGURE 12. A physical model for ejecta formation: half of the problem is shown. Here $v(t)$ is the drop velocity, $V(t)$ the velocity of the ejecta, $\Delta y$ the penetration of the drop into the film, $r_{0}$ the drop radius, $h_{\mathrm{e}}$ the ejecta thickness, $R(t)$ the rim radius, and $\theta(t)$ the angle of the ejecta.

[38], and is not intended to be an accurate description of the drop splash problem in all possible regimes of splashes (e.g. on very thin films and solid substrates).

In order to develop stability analysis of the drop splash problem in the regime when its base state and the domain are time-dependent, which is especially the case for early times of the impact, let us consider the geometry of the problem as shown in figure 7 the ejecta of time-dependent radius $R(t)$, time-dependent curvature of the rim of the ejecta $a^{-1}(t) \gg R^{-1}(t)$, and time-dependent acceleration $g(t)=V^{\prime}(t)$. Then, based on the understanding developed in $\S 2.1$ the model (2.6) with $n=0$ can be generalized so that the evolution of the amplitude $f_{m}$ of the discrete wavelength $\lambda=2 \pi R / m$, i.e. $k=m / R$, obeys

$$
\frac{\mathrm{d}^{2} f_{m}}{\mathrm{~d} t^{2}}+\frac{|m|}{R(t)}\left[\frac{m^{2}}{B o} \frac{1}{R^{2}(t)}-g(t)\right] f_{m}=0,
$$

where $B o=\rho g_{0} R_{0}^{2} / \sigma$, and the time-dependent functions $g(t), R(t)$, and $a(t)$ are independent of each other and to be constructed below. This model will be utilized in our subsequent studies of stability on time-dependent domain. The construction below will justify the power laws for the time-dependent functions in (3.1) to be used later.

It should be emphasized that the model (3.1) is derived phenomenologically by generalizing (2.6) rather then linearizing the Navier-Stokes equations around the time-dependent base state systematically, which is impeded by the complex time-dependent geometry of the problem. Even in simpler problems such as excitation of surface waves in a closed cylindrical container, which is subject to vertical oscillations, the complexity of the problem forces one to make numerous approximations [43].

3.1.1. Simplifying assumptions. Let a crossection (along the axis of symmetry) of the axisymmetric problem of the drop impact on a film of the same liquid be as shown in figure 12 ; suppose that the drop radius is $r_{0}$. Since we are concerned with early stages of the drop impact, we will invoke a number of simplifying assumptions and facts:

- The drop is circular, and the velocity of the drop is uniform throughout the drop prior to impact, $t<0$, with the value $v_{0}$. Also, the impact is energetic (as opposed to drop spreading), which corresponds to substantial values of the drop release height, and thus 
the penetrating drop retains its shape during early stages of the impact. These are the regimes explored experimentally in [38].

- The ejecta is formed from the underlying liquid layer but not the drop fluid, as was proved experimentally by coloring the film fluid to distinguish it from the drop fluid [62], where it was also shown that the initial ejecta speed can be more than 10 times the impact velocity of the drop $v_{0}$. Furthermore, the ejecta inclination is assumed to be always tangent to the drop circumference at the point $A$ of intersection with the film, cf. figure 12 , and moving as a single whole with the same velocity $V(t)$.

- The hydrostatic energy due to gravity in this system can be neglected because, for example, for a water drop of radius $r_{0}=10^{-3} \mathrm{~m}, v_{0}=1 \mathrm{~m} / \mathrm{s}$, its kinetic energy $\sim$ $\rho\left(v_{0}^{2} / 2\right)(4 \pi / 3) r_{0}^{3} \sim 10^{-6} \mathrm{~N} \cdot \mathrm{m}$, potential energy due to surface tension is $\sim 4 \pi r_{0}^{2} \sigma \sim$ $10^{-6} \mathrm{~N} \cdot \mathrm{m}$, while hydrostatic potential energy change over the characteristic depth of penetration $\sim r_{0}$ is $\sim \rho g(4 \pi / 3) r_{0}^{4} \sim 5 \cdot 10^{-8} \mathrm{~N} \cdot \mathrm{m}$, i.e. much smaller than kinetic and surface tension energies.

3.1.2. General relations. Given the above simplifying assumptions, the following considerations based on the geometry of the problem and basic conservation laws lead to a simple model for the ejecta evolution.

Geometric considerations. The distance traveled by the drop (and its tip) from the moment of impact is $\Delta y=\int_{0}^{t} v(t) \mathrm{d} t$, which approximately equals $v(t) t$, as the velocity of the drop changes insignificantly over small time interval $t$ following the moment of impact at $t=0$. From geometric considerations,

$$
\Delta y=r_{0}(1-\cos \theta) \simeq r_{0}\left(\frac{\theta^{2}}{2}-\frac{\theta^{4}}{24}\right),
$$

as the angle $\theta$ is small for early times. Using the formula for $\Delta y$ we find the drop velocity

$$
v(t)=\frac{r_{0}}{t}(1-\cos \theta) \simeq \frac{r_{0}}{t}\left(\frac{\theta^{2}}{2}-\frac{\theta^{4}}{24}\right) .
$$

Since as $t \rightarrow+0, v \rightarrow v_{0}$, we get the time-dependence of the angle $\theta$ and the velocity:

$$
\theta(t)=\sqrt{\frac{2 v_{0}}{r_{0}}} t^{1 / 2}, v(t)=v_{0}-\frac{v_{0}^{2}}{6 r_{0}} t+\ldots
$$

respectively.

Mass conservation. Based on the experimental observation that ejecta is formed from the film phase [62], the volume of the penetrated portion of the drop (shaded segment in figure 12) should be equal to that of the formed ejecta (also shaded in figure 12),

$$
\Delta_{\text {segm }}=\Delta_{\mathrm{e}},
$$

where the volume of the shaded segment is $\Delta_{\text {segm }}=\pi(\Delta y)^{2}\left(3 r_{0}-\Delta y\right) / 3 \simeq \pi r_{0}(\Delta y)^{2}$ for small $\Delta y$. The volume of the ejecta is also straightforward to compute from the geometry of the problem, cf. figure $12, \Delta_{\mathrm{e}} \simeq \pi h_{\mathrm{e}}(R-r)(R+r)$ for small $\Delta y$, where $h_{\mathrm{e}}$ is the ejecta thickness, $R$ the radius of the rim (i.e. the distance from the axis of symmetry to the tip of the ejecta), and $r(t)=r_{0} \sin \theta$ the radius of the base of the penetrated segment.

Momentum balance. Due to the fact that the drop is liquid and thus deforms in the process of impact, the application of momentum balance is less straightforward. The decrease in the $y$-momentum of some unknown portion $\Delta$ of the drop mass is equal to the increase in the $y$-component of the ejecta momentum

$$
\rho \Delta\left(v(t)-v_{0}\right)=\rho \Delta_{\mathrm{e}} V(t) \sin \theta,
$$


where $V(t)$ is the velocity of the ejecta. The value of $\Delta$ needs to be found as a part of the solution. Note that because of the symmetry of the problem the total radial momentum of the ejecta stays zero. In general, one needs to account for the added mass effect due to the presence of the pre-existing film similar to the water hammering phenomenon [57], which may be taken care of by introducing a factor in front of $v(t)$ on the left hand side of (3.6). Here it is taken to be one, but in general it depends on the film thickness $h_{\text {film }}$.

Energy balance. The initial energy of the system - the sum of the kinetic energy of the relevant portion $\Delta$ of the drop mass, $\rho \Delta v_{0}^{2} / 2$, the potential surface energy of the drop, $4 \pi r_{0}^{2} \sigma$, and the relevant potential surface energy of the film, $\pi[r(t)+l(t)]^{2} \sigma$, where $l=h_{\mathrm{e}} / \sin \theta \simeq h_{\mathrm{e}} / \theta$ :

$$
E_{0}=\rho \Delta \frac{v_{0}^{2}}{2}+4 \pi r_{0}^{2} \sigma+\pi[r(t)+l(t)]^{2} \sigma,
$$

should be equal to the energy of the system $E(t)$ at later times, $t>0$, i.e.

$$
E_{0}=E(t) \text {. }
$$

For small times,

$$
E(t)=\rho \Delta \frac{v^{2}}{2}+\left[4 \pi r_{0}^{2}-\pi\left(2 r_{0} \Delta y\right)\right] \sigma+\left[\rho \Delta_{\mathrm{e}} \frac{V^{2}}{2}+2 \pi(R-r)(R+r) \sigma\right],
$$

which consists of the kinetic energy and the reduction in the potential surface energy of the drop, as well as the kinetic and potential energies of the ejecta. Note that $2 \pi(R-r)(R+r)$ is the general total external (exposed) area of the ejecta for small $\Delta y$.

Given the above considerations, the unknowns are $\Delta(t), v(t), V(t), \theta(t), R(t)$, and $h_{\mathrm{e}}(t)$, while the number of relations (3.2) (3.5), (3.6), and (3.8) is four. One may also invoke angular momentum conservation, but it turns out that for small times this is not necessary as the geometric relation (3.2) gives information about both $\theta(t)$ and $v(t)$ found to be given by (3.4).

3.1.3. Small time limit analysis. The mass conservation (3.5) gives

$$
\pi r_{0}^{3} \frac{\theta^{4}}{4}=\pi h_{\mathrm{e}}(R-r)(R+r)
$$

which implies that since $r \sim \theta$, then $R \sim \theta$ and therefore $h_{\mathrm{e}} \sim \theta^{2}$, which is also in agreement with the expectation that the ejecta is thin compared to its radius $R$.

In the limit of small times $t \rightarrow+0$, the ejecta velocity $V(t)$ is determined from the momentum conservation (3.6)

$$
V(t)=-v_{0} \frac{\Delta}{3 \pi r_{0}^{3}} \theta^{-3}
$$

where the portion of the drop mass $\Delta$, which changes its momentum at the early stage of the impact, is to be determined from the energy balance (3.8)

$$
\overbrace{\rho \Delta\left(v_{0}^{2}-v^{2}\right) / 2}^{\sim \Delta \theta^{2}}+\overbrace{\pi(r+l)^{2} \sigma}^{\theta^{2}}=-\overbrace{2 \pi r_{0} \Delta y \sigma}^{\theta^{2}}+\overbrace{\rho \Delta_{\mathrm{e}} V^{2} / 2}^{\Delta^{2} \theta^{-2}}+\overbrace{2 \pi(R-r)(R+r) \sigma}^{\theta^{2}},
$$

where we indicated the order of magnitude of each term via $\theta \rightarrow 0$ as $t \rightarrow 0$. With the goal to balance orders, let us suppose $\Delta \sim \theta^{\alpha}$, which gives two options:

$\alpha=4: V \sim \theta$, which implies that the surface tension and kinetic energies are decoupled and the balance is achieved in each category of energies, i.e. the surface tension and the kinetic energies balance each other independently (the kinetic energy of ejecta comes from that of the drop). 
$\alpha=2: V \sim \theta^{-1}$, which implies that the kinetic energy of the ejecta comes from the potential energy of the surface, but the kinetic energy of the drop portion is negligible.

As known experimentally from the kinematics of the ejecta [38], the velocity of the ejecta first rapidly increases from zero value and then decreases due to surface-tension retraction effect. Therefore, the case $\alpha=4$ corresponds to the very early times of the impact, while the case $\alpha=2$ is valid for later but still small times.

The above model shows that the power-law approximations for the ejecta acceleration and the geometry of the rim for early times are reasonable: this fact will be utilized in the subsequent model (3.11) based on (3.1). As discussed in §3.1.1, this model is derived from a number of simplifying assumptions. One can enhance the present model, e.g. by (a) relaxing the mass conservation condition (3.5), and (b) accounting for the part of the drop energy dissipated in the film.

3.2. Linear stability picture. Based on the above discussion we may assume that all time-dependent terms in the model (3.1) are of power-law form. Let us also suppose that $g=\widehat{g} R^{-2}, \widehat{g}=$ const, which allows us to rewrite (3.1) (dropping indexes) as

$$
t^{a} f^{\prime \prime}-c f=0, c=\widehat{c}|m|\left[g-\frac{m^{2}}{B o}\right]>0, \widehat{c}=\text { const. }
$$

Letting $f=\sqrt{c t} \phi(\tau), \tau=\chi t^{b}$, equation (3.11) is transformed to

$$
\tau^{2} \phi^{\prime \prime}+\tau \phi^{\prime}-b^{-2}\left[c t^{2-a}+\frac{1}{4}\right] \phi=0,
$$

which with the choice $b=(2-a) / 2, a \neq 2^{6}$, and $\chi=2 \sqrt{c} /(2-a)$ becomes the modified Bessel equation [1]

$$
\tau^{2} \phi^{\prime \prime}+\tau \phi^{\prime}-\left(\tau^{2}+\nu^{2}\right) \phi=0, \nu=(2-a)^{-1} .
$$

Its solutions read

$$
\phi= \begin{cases}C_{1} I_{\nu}(\tau)+C_{2} I_{-\nu}(\tau), & \nu \notin \mathbb{Z} \\ C_{1} I_{\nu}(\tau)+C_{2} K_{\nu}(\tau), & \nu \in \mathbb{Z}\end{cases}
$$

where $C_{1,2}$ are constants, and $I_{\nu}(\tau)$ and $K_{\nu}(\tau)$ are the Bessel functions of the first and second kind, respectively. The asymptotic behaviors of $I_{\nu}(\tau)$ and $K_{\nu}(\tau)$ for $\tau \rightarrow \infty$ are given by [1]

$$
\begin{aligned}
I_{\nu}(\tau) & =\frac{e^{\tau}}{\sqrt{2 \pi \tau}}\left(1+O\left(\tau^{-1}\right)\right), \\
K_{\nu}(\tau) & =\sqrt{\frac{\pi}{2 \tau}} e^{-\tau}\left(1+O\left(\tau^{-1}\right)\right) .
\end{aligned}
$$

Therefore, if we let $0<a<2$ so that $b>0$ and thus $\chi>0$, we find that $t \rightarrow+\infty$ when $\tau \rightarrow+\infty$ and

$$
f \sim t^{a / 4} \exp \left[\frac{2 \sqrt{c}}{2-a} t^{\frac{2-a}{2}}\right]
$$

\footnotetext{
${ }^{6}$ The case $a=2$ gives $f=t^{2}$ provided $c=2$.
} 
which implies that the largest growth rate corresponds to the largest $c$ similar to the timeindependent case $^{7}$ !

If $g(t)$ has a time-rate different from that for $R^{-2}(t)$, then either the sign of the coefficient $c$ in the model (3.11) is changed or $c$ has no extrema. Since the latter case does not lead to a finite critical wavenumber selection, we are left to consider the former case:

$$
t^{a} f^{\prime \prime}+c f=0, c>0,
$$

the solution of which is also sought in the form $f=\sqrt{c t} \phi(\tau), \tau=\chi t^{b}$ with $b=(2-a) / 2$. As a result, equation (3.17) reduces to the standard Bessel equation

$$
\tau^{2} \phi^{\prime \prime}+\tau \phi^{\prime}+\left(\tau^{2}-\nu^{2}\right) \phi=0, \nu=(2-a)^{-1},
$$

all solutions of which are decaying in time for both integer and non-integer $\nu$, e.g. for $\nu \notin \mathbb{Z}$ :

$$
\phi \sim \sqrt{\frac{2}{\pi \tau}}\left[C_{1} \cos \left(\tau-\frac{\nu \pi}{2}-\frac{\pi}{4}\right)+C_{2} \sin \left(\tau-\frac{\nu \pi}{2}-\frac{\pi}{4}\right)\right]+O\left(\tau^{-3 / 2}\right) .
$$

Thus, motivated by conjecture 1 , we arrive at the following result in the framework of the models (2.6) and (3.1).

THEOREM 1. If an instability pattern with a finite critical wavenumber is observed in the system (3.1) on time-dependent domain, then the critical wavenumber of this pattern can be determined from the model (2.6) on time-invariant domain with an appropriately redefined Bond number.

Long-time simulations of (3.1) confirm this theoretical prediction, i.e. the case $g \sim$ $R^{-2}$ gives the long-time behavior which agrees with that in the time-independent case. The above theorem is also consistent with the experimental observation that the number of spikes is constant even though the base state and the domain are evolving.

\section{Conclusions}

The offered linear models (2.6) and (3.1), despite their simplicity, provide some insight into the fundamental physical processes in the drop splash problem for certain ranges of physical parameters [38] and also capture the key dynamical features - regular and frustrated regimes - in the crown formation. Further exploration is needed to uncover other possible dynamical effects, e.g. insertion or disappearance of spikes as the crown evolves in time, which can be due to Eckhaus instability [17], as well as characterization of transitions between regular and irregular (possibly chaotic) stability patterns.

Nonlinear formulation of the drop splash phenomena will naturally require one to consider it as a dynamic bifurcation problem since the parameters $R(t), a(t)$, etc. are time-dependent. Also, the time-dependent geometry - the rim radius $R(t)$ and curvature $a(t)$ in (3.11) - were considered here as (external) variables, which is appropriate at the linear level of description. However, in the full nonlinear problem formulation, both $R(t)$ and $a(t)$ are internal to the drop splash dynamics, as they are determined as a part of the

\footnotetext{
${ }^{7}$ Note that the derived asymptotics for $t \rightarrow+\infty$ can also be found with the help of the WKBJ method [4], i.e. first by transforming (3.11) via $f=t \phi(\tau)$ with $\tau=t^{-1}$ and then looking for the solution of the transformed equation $\tau^{4-a} \phi^{\prime \prime}-c \phi=0$ in the form $\phi=e^{S(\tau)}$. The latter leads to the equation $\left(S^{\prime}\right)^{2}+S^{\prime \prime}=c \tau^{a-4}$. With the assumption $\left(S^{\prime}\right)^{2} \gg S^{\prime \prime}$, which is justified a posteriori, to the leading order $S(\tau)= \pm \sqrt{c} \frac{2}{2-a} \tau^{\frac{a-2}{2}}$, so that indeed $S^{\prime \prime} /\left(S^{\prime}\right)^{2}= \pm \frac{1}{\sqrt{c}} \frac{a-4}{2} \tau^{\frac{2-a}{2}} \rightarrow 0$ as $\tau \rightarrow 0$. Corrections terms to $S(\tau)$, i.e. $S(\tau)=$ $\sqrt{c} \frac{2}{2-a} \tau^{\frac{a-2}{2}}+C(\tau)$, are found similarly and lead to (3.16).
} 
solution similar to the domain size in the Stefan problem [13], for example. All these subtleties in the dynamic behavior of the crown formation can be unraveled only with the help of a systematic reduction of the Navier-Stokes equations to a simple low-dimensional dynamical model.

The above considerations also bring up questions of general character such as (a) timedependent symmetries and proper language for them $[\mathbf{5}, \mathbf{5 4}]$, and (b) weakly nonlinear theory for time-dependent spatial domains with symmetries.

\section{Appendix A. Richtmyer-Meshkov instability with a nearly impulsive acceleration}

In this Appendix we show that strictly impulsive acceleration is not necessary for the Richtmyer-Meshkov instability to take place, but instead only a relatively sharp change in the interfacial velocity suffices.

Consider the equation governing linear perturbation $f(t)$ of the interface location relative to a (flat) reference state

$$
\frac{\mathrm{d}^{2} f}{\mathrm{~d} t^{2}}=a(t) f(t)
$$

which is the starting point of the Richtmyer analysis [50]. Let the acceleration $a(t)$ be of delta-sequence $\delta_{\epsilon}(t)^{8}$ form rather than the Dirac delta-function $\delta(t)$. While there are many delta-sequences, for the purpose of integrating equation (A.1) it is convenient to consider the form of $\delta_{\epsilon}(t)$, which is continuous in time, e.g.

$$
\delta_{\epsilon}(t)=\frac{\epsilon}{\pi\left(t^{2}+\epsilon^{2}\right)} .
$$

Then, the equation has the solution

$$
f(t)=C_{1} \operatorname{ch} \sqrt{\eta}+C_{2} \operatorname{sh} \sqrt{\eta}, \eta=\frac{\epsilon}{\pi} t^{2} .
$$

For $\epsilon \ll 1$ and $t \leq O(1)$ :

$$
f(\eta) \sim \text { const }_{1}+\text { const }_{2} t,
$$

i.e. the interface is linearly algebraically unstable, as in the ideal Richtmyer-Meshkov case when the acceleration is the delta function. This result is, of course, intuitive since otherwise the Richtmyer-Meshkov instability would not be a robust phenomenon.

One can also use a discontinuous delta-sequence:

$$
a(t)=\left\{\begin{array}{l}
0, t<-\frac{1}{2 n} \\
n,-\frac{1}{2 n}<t<\frac{1}{2 n}, \\
0, t>\frac{1}{2 n}
\end{array}\right.
$$

so that for $t>1 / 2 n$ :

$$
f^{\prime}(t)=f(0)+O\left(n^{-2}\right), n \gg 1 .
$$

Clearly, the interface is linearly algebraically unstable for this case as well.

Acknowledgements. The author is grateful to Professor Edgar Knobloch for insightful and encouraging discussions.

\footnotetext{
${ }^{8}$ By definition, $\lim _{\epsilon \rightarrow+0}\left(\delta_{\epsilon}, \phi\right)=\phi(0)=(\delta, \phi)$ for any $\phi$, where $\phi \in D$ and $\delta_{\epsilon} \in D^{\prime}$.
} 


\section{References}

[1] M. Abramowitz and I. A. Stegun, Handbook of mathematical functions: with formulas, graphs, and mathematical tables, Dover Publications, 1965.

[2] A. Armaou and P. D. Christofides, Finite-dimensional control of nonlinear parabolic PDE systems with time-dependent spatial domains using empirical eigenfunctions, Int. J. Appl. Math. Comput. Sci. 11 (2001), 287-317.

[3] D. Armbruster, J. Guckenheimer, and P. Holmes, Heteroclinic cycles and modulated travelling waves in systems with $O(2)$ symmetry, Physica D 29 (1988), 257-282.

[4] C. M. Bender and S. A. Orzsag, Advanced mathematical methods for scientists and engineers: asymptotic methods and perturbation theory, Springer, 1999.

[5] W.-J. Beyn and V. Thümmler, Freezing solutions of equivariant evolution equations, SIAM J. Appl. Dyn. Syst. 3 (2004), 85-116.

[6] F. Borgnis and C. H. Papas, Electromagnetic waveguides and resonators, Lecture Notes, California Institute of Technology, Pasadena, CA, 1972.

[7] C. C. Chow, A. Bers, and A. K. Ram, Spatiotemporal chaos in the nonlinear three-wave interaction, Phys. Rev. Lett. 68 (1992), 3379-3382.

[8] G. E. Cossali, M. Marengo, A. Coghe, and S. Zhdanov, The role of time in single drop splash on thin film, Exp. Fluids 36 (2004), 888-900.

[9] J. D. Crawford, E. Knobloch, and H. Riecke, Period-doubling mode interactions with circular symmetry, Physica D 44 (1990), 340-396.

[10] G. Dangelmayr, Steady-state mode interactions in the presence of $O(2)$ symmetry, Dyn. Stab. Sys. 1 (1986), $159-185$.

[11] G. Dangelmayr and E. Knobloch, Hopf bifurcation with broken circular symmetry, Nonlinearity 4 (1991), 399-427.

[12] S. H. Davis, The stability of time-periodic flows, Ann. Rev. Fluid Mech. 8 (1976), 57-74.

[13] S. H. Davis, U. Müller, and C. Dietsche, Pattern selection in single-component systems coupling Bénard convection and solidification, J. Fluid Mech. 144 (1984), 133-151.

[14] J. Dittrich, P. Duclos, and N. Gonzalez, Stability and instability of the wave equation solutions in a pulsating domain, Rev. Math. Phys. 10 (1998), 925-962.

[15] V. V. Dodonov, A. B. Klimov, and D. E. Nikonov, Quantum particle in a box with moving walls, J. Math. Phys. 34 (1993), 3391-3404.

[16] P. G. Drazin and W. H. Reid, Hydrodynamic stability, Cambridge University Press, Cambridge, 2004.

[17] W. Eckhaus, Studies in non-linear stability theory, Springer-Verlag, New York, 1965.

[18] _ On modulation equations of the Ginzburg-Landau type, Proceedings of the second international conference on industrial and applied mathematics (Jr. R. E. O’Malley, ed.), SIAM, Philadelphia, 1991, pp. 83-98.

[19] _. The Ginzburg-Landau manifold is an attractor, J. Nonlinear Sci. 3 (1993), 329-348.

[20] O. G. Engel, Crater depth in fluid impacts, J. Appl. Phys. 37 (1966), 1798-1808.

[21] _ Initial pressure, initial flow velocity, and the time dependence of crater depth in fluid impacts, J. Appl. Phys. 38 (1967), 3935-3940.

[22] B. F. Farrell and P. J. Ioannou, Generalized stability theory. Part II: Nonautonomous operators, J. Atmos. Sci. 53 (1996), 2041-2053.

[23] __ Perturbation growth and structure in time-dependent flows, J. Atmos. Sci. 56 (1999), 3622-3639.

[24] M. A. Fernández and P. Le Tallec, Linear stability analysis in fluid-structure interaction with transpiration. Part I: Formulation and mathematical analysis, Comp. Meth. Appl. Mech. Engr. 192 (2003), 4805-4835.

[25] J. Ferreira, R. Benabidallah, and J. E. Muñoz Rivera, Asymptotic behaviour for the nonlinear beam equation in a time-dependent domain, Rend. Mat. Appl. 19 (1999), 177-193.

[26] M. G. Floquet, Sur les équations différentielles linéaires à coefficients périodiques, Ann. École Norm. Sup. 12 (1883), 47-88.

[27] H. Fujita and N. Sauer, On existence of weak solutions of the Navier-Stokes equations in regions with moving boundaries, J. Fac. Sci. Univ. Tokyo Sec. IA 17 (1970), 403-420.

[28] J. M. Fullana and S. Zaleski, Stability of a growing end rim in a liquid sheet of uniform thickness, Phys. Fluids 11 (1999), 952-954.

[29] J. Gjorgjieva and J. Jacobsen, Turing patterns on growing spheres: the exponential case, Discr. Cont. Dyn. Sys. Suppl. (2007), 436-445.

[30] D. Gueyffier and S. Zaleski, Formation de digitations lors de l'impact d'une goutte sur un film liquide, C. R. Acad. Sci. Paris, S'erie II 326 (1998), 839-844. 
[31] G. M. Homsy, Global stability of time-dependent flows: impulsively heated or cooled fluid layers, J. Fluid Mech. 60 (1973), 129-139.

[32] G. Iooss and J. Los, Bifurcation of spatially quasi-periodic solutions in hydrodynamic stability problems, Nonlinearity 3 (1990), 851-871.

[33] M. H. Jensen and P. Bak, Spatial chaos, Phys. Scripta 9 (1985), 64-69.

[34] E. Knobloch and J. Guckenheimer, Convective transitions induced by a varying aspect ratio, Phys. Rev. A 27 (1983), 408-417.

[35] S. Kondo and R. Asai, A reaction-diffusion wave on the skin of the marine angelfish Pomacanthus, Nature 376 (1995), 765-768.

[36] R. Krechetnikov, Rayleigh-Taylor and Richtmyer-Meshkov instabilities of flat and curved interfaces, J. Fluid Mech. 625 (2009), 387-410.

[37] _ Stability of liquid sheet edges, Phys. Fluids 22 (2010), 092101.

[38] R. Krechetnikov and G. M. Homsy, Crown-forming instability phenomena in the drop splash problem, J. Colloid Interface Sci. 331 (2009), 555-559.

[39] K. Lee, A mixed problem for hyperbolic equations with time dependent domains, J. Math. Anal. Appl. 16 (1966), 445-471.

[40] J. L. Lions, Singular perturbations and some non linear boundary value problems, MRC Tech. Summary Rep. 421, Univ. Wisconsin, 1963.

[41] W. C. Macklin and G. J. Metaxas, Splashing of drops on liquid layers, J. Appl. Phys. 47 (1976), 3963-3970.

[42] A. Madzvamuse, E. A. Gaffney, and P. K. Maini, Stability analysis of non-autonomous reaction-diffusion systems: the effects of growing domains, J. Math. Biol. 61 (2010), 133-164.

[43] E. Meron and I. Procaccia, Low-dimensional chaos in surface waves: theoretical analysis of an experiment, Phys. Rev. A 34 (1986), 3221-3237.

[44] T. Miyakawa and Y. Teramoto, Existence and periodicity of weak solutions of the Navier-Stokes equations in a time dependent domain, Hiroshima Math. J. 12 (1982), 513-528.

[45] W. Pesch and L. Kramer, General mathematical description of pattern-forming instabilities, Pattern formation in liquid crystals (A. Buka and L. Kramer, eds.), Springer-Verlag, New Yark, 1995, pp. 69-90.

[46] J. Porter and E. Knobloch, Complex dynamics in the $1: 3$ spatial resonance, Physica D 143 (2000), 138168.

[47] J. Prat, I. Mercader, and E. Knobloch, Resonant mode interaction in Rayleigh-Bénard convection, Phys. Rev. E 58 (1998), 3145-3156.

[48] C. G. R. and A. A. Mizoni, An asymptotic solution for the wave equation in a time-dependent domain, SIAM Rev. 23 (1981), 1-9.

[49] L. Rayleigh, Investigation of the character of the equilibrium of an incompressible heavy fluid of variable density, Proc. London Math. Soc. 14 (1883), 170-177.

[50] R. D. Richtmyer, Taylor instability in shock acceleration of compressible fluids, Comm. Pure Appl. Math. XIII (1960), 297-319.

[51] R. Rioboo, C. Bauthier, J. Conti, M. Voué, and J. De Coninck, Experimental investigation of splash and crown formation during single drop impact on wetted surfaces, Exp. Fluids 35 (2003), 648-652.

[52] E. D. Rogak, A mixed problem for the wave equation in a time dependent domain, Arch. Rat. Mech. Anal. 22 (1966), 24-26.

[53] I. V. Roisman and C. Tropea, Impact of a drop onto wetted wall: description of crown formation and propagation, J. Fluid Mech. 472 (2002), 373-397.

[54] C. W. Rowley, I. G. Kevrekidis, J. E. Marsden, and K. Lust, Reduction and reconstruction for self-similar dynamical systems, Nonlinearity 16 (2003), 1257-1275.

[55] G. Schneider, The validity of generalized Ginzburg-Landau equations, Math. Methods Appl. Sci. 19 (1996), 717-736.

[56] Bifurcation theory for dissipative systems on unbounded cylindrical domains - an introduction to the mathematical theory of modulation equations, Z. Angew. Math. Mech. 8 (2001), 507-522.

[57] L. I. Sedov, Similarity and dimensional methods in mechanics, Academic Press, New York, 1961.

[58] J. Serrin, On the stability of viscous fluid motion, Arch. Rat. Mech. Anal. 3 (1959), 1-13.

[59] M. Shahinpoor and G. Ahmadi, Stability of Cosserat fluid motions, Arch. Rat. Mech. Anal. 47 (1972), $188-194$.

[60] G. I. Taylor, The instability of liquid surfaces when accelerated in a direction perpendicular to their planes. I. Waves on fluid sheets, Proc. R. Soc. London, Ser. A 201 (1950), 192-196.

[61] Y. Teramoto, On the stability of periodic solutions of the Navier-Stokes equations in a noncylindrical domain, Hiroshima Math. J. 13 (1983), 607-625.

[62] S. T. Thoroddsen, The ejecta sheet generated by the impact of a drop, J. Fluid Mech. 451 (2002), 373-381. 
[63] M. F. Trujillo and C. F. Lee, Modeling crown formation due to the splashing of a droplet, Phys. Fluids 13 (2001), 2503-2516.

[64] A. van Harten, On the validity of the Ginzburg-Landau equation, J. Nonlinear Sci. 1 (1991), 397-422.

[65] A.-B. Wang and C.-C. Chen, Splashing impact of a single drop onto very thin liquid films, Phys. Fluids 12 (2000), 2155-2158.

[66] A. M. Worthington, A study of splashes, Longmans, London, 1908.

[67] A. L. Yarin, Drop impact dynamics: splashing, spreading, receding, bouncing, ..., Annu. Rev. Fluid Mech. 38 (2006), 159-192.

[68] L. V. Zhang, P. Brunet, J. Eggers, and R. D. Deegan, Wavelength selection in the crown splash, Phys. Fluids 22 (2010), 122105.

Department of Mechanical Engineering, University of California, SANTA Barbara, CA 93106, USA

E-mail address: rkrecheteengineering.ucsb.edu 